\title{
Effect of the error estimation of nodes in the cluster formation phase in wireless sensor networks with adaptive transmission probability
}

\author{
Edgar Romo ${ }^{1}$, Mario Rivero ${ }^{2}$, Iclia Villordo ${ }^{3}$ \\ 1 UPIITA - IPN, Av. Instituto Politécnico Nacional 2580, \\ Barrio La Laguna Ticomán, Gustavo A. Madero, 07340, México .D.F. \\ eromom0900@alumno.ipn.mx \\ 2 CIC - IPN, Av. Juan de Dios Bátiz, Esq. Miguel Othón de Mendizábal, \\ Nueva Industrial Vallejo, Delegación Gustavo A. Madero, 07738, México D.F. \\ mriveroadipn.mx \\ 3 UPIITA - IPN, Av. Instituto Politécnico Nacional 2580, \\ Barrio La Laguna Ticomán, Gustavo A. Madero, 07340, México .D.F. \\ ivillordodipn.mx
}

\begin{abstract}
In this paper, the performance of a cluster-based wireless sensor network is studied when an adaptive transmission probability scheme is used to form the clusters. The cluster scheme is based on the well-known LEACH protocol, which organizes the nodes inside a wireless sensor network in clusters in order to distribute the energy load of each node. However, unlike LEACH, nodes are chosen to be cluster heads or cluster members only based on the order of arrival of nodes (called LEACH MOD), instead of whether or not a node has been a cluster head previously, in order to reduce the processing time and energy consumption added by the role selection scheme proposed by LEACH. The nodes transmit with a certain probability in order to create the cluster; as such, the adaptive transmission scheme, chooses the adequate value for the transmission probability based on the estimation of a number of nodes attempting a transmission in the following time slots. In this work, we study the impact of the error estimation on the energy consumption and the average formation cluster time when the network works with such scheme.
\end{abstract}

Keywords: Wireless Sensor Network, adaptive transmission probability, LEACH, energy consumption, clustering formation.

\section{Introduction}

The growing interest in control, knowledge and monitoring all sorts of physical variables around us causes the need to find new technologies that simplify such task. In order to perform such tasks, there are different techniques that allow doing that environmental sensing, from human supervision to technologies like closed circuit video surveillance, process automation or the use of sensor networks.

In this case, the use of a Wireless Sensor Network (WSN) gives the possibility to collect different information depending on the application, providing the possibility of 
constant monitoring. Additionally, WSNs offer the advantage of the use of small nodes that can be installed inside different spaces, like a wall, a roof, under the pavement, etc. However, energy consumption becomes a focal parameter because the nodes, due to their small size, are provided with a battery of small size and capacity.

Efficient energy consumption inside the network allows extending the lifetime of the system. When a node drains all its battery, this node no longer operates in the network and all the nodes associated to it and the whole system suffers an operational degradation, due to information losses. Specifically, the effects in the system when a node consumes its battery energy are:

- Information loss.

- Reduction in the coverage area.

- Connection losses among nodes.

The energy consumption is even more critical in such scenarios where the coverage area is particularly high, such as monitoring in a desert, forest, or any other open place. In this case, the energy consumption increases due to the high-energy transmissions of nodes in order to reach the sink node. In this case the network have two options to cover the total coverage area:

- Increase the total number of nodes in the network

- Increase the distance between each node

This work focuses on such environments. Specifically, a clustering protocol is proposed, based on the well-known LEACH [1] protocol, in order to allow efficient network operation in large area. To this end we propose LEACH-MOD, which establishes a modification in the cluster formation phase as follows: unlike LEACH, nodes are chosen to be cluster head or cluster members only based on the order of arrival of the nodes in order to reduce the processing time and the energy consumption.

Furthermore, nodes do not transmit in the cluster formation phase with a constant transmission probability $\tau$, but rather this value varies according to the number of nodes remaining to transmit their control packet. As such, the transmission probability decreases in each successful transmission. According to the results presented in this work, this adaptive transmission probability reduces both energy consumption and cluster formation time.

\section{System Model}

In this section, the main system parameters and assumptions are presented. A square coverage area of 100 squared meters with a variable number of nodes is considered in order to analyze the performance of the cluster formation process under different scenarios. 
Effect of the error estimation of nodes in the cluster formation phase in wireless sensor networks ...

The energy consumption model considered is now described in detail. We considered normalized energy units as values of energy required for the packet transmission and reception. This is basically in order to have a general energy consumption model that can be easily scaled to any commercial equipment. Building on this, we assume the following:

- The energy required to transmit a packet in the cluster formation phase $(\mathrm{CF})$, is $E_{t x}^{C F}=0.02$ units.

- The energy required to receive a packet in the cluster formation phase is $E_{r x}^{C F}$ $=0.01$ units.

- The energy required to transmit a packet from a cluster member to a cluster head in the steady state is $E_{t x}^{\text {Steady }}=0.01$ units.

- The energy consumed by the cluster head in order to receive a packet from a cluster member in the steady state is $E_{r x}^{\text {steady }}=0.01$ units.

- The energy consumed by the cluster head to transmit a packet to the sink node $(\mathrm{CH} \rightarrow \operatorname{Sink})$, is $E_{t x}^{C H}>\operatorname{Sink}=0.2$ units.

Note that the energy required to transmit a packet is always higher than the energy required to receive such packet. Also the packet transmission from the cluster head to the sink node is the most energy consuming.

Finally we consider a percentage of nodes that act as cluster heads as follows: for $\mathrm{LEACH}$, five percent of nodes in average act as cluster heads while in LEACH-MOD, $N_{C H}$ nodes always become cluster heads.

\section{LEACH}

LEACH is a protocol that organizes the nodes inside a wireless sensor network in clusters in order to distribute the energy load of each node. In this way, all nodes organize themselves to take part in a unique cluster as cluster head $(\mathrm{CH})$ or as cluster member (CM). The cluster head of each cluster have the function of gather the sensed data of each node that belongs to the cluster. When the cluster head has gathered all the cluster information, it sends the data to the sink or base station.

We can easily see that $\mathrm{CHs}$ consume much more energy since they have to be constantly receiving data from the CMs, and perform high-energy transmissions to the sink node. Hence, $\mathrm{CHs}$ drain their energy faster. For that reason, LEACH rotates the function of cluster heads among all nodes in the network in order to balance the energy consumption equitably. This rotation occurs periodically. The LEACH protocol considers 20-second TDMA periods, after which clusters are broken and the cluster formation starts again selecting new $\mathrm{CHs}$. 
This protocol is composed by two phases: The cluster formation phase, where nodes are selected either as $\mathrm{CHs}$ or CMs and the steady state phase, where clusters are already established and data transmission is achieved. In the former phase, a random access protocol is used. Specifically, the slotted NP-CSMA scheme is applied, where nodes transmit in each time slot with probability $\tau$. In the steady state phase, a TDMA based scheme is preferred since the $\mathrm{CH}$ already knows the number of nodes that will report data in the cluster. Hence, transmissions can be done in an orderly fashion. Nodes will choose to become cluster heads according to the following equation [2]:

$$
T(n)=\left\{\begin{array}{cc}
\frac{P}{1-P x\left(r \bmod \frac{1}{P}\right)} & \text { if } n \in G \\
0 & \text { otherwise }
\end{array}\right.
$$

Where $P$ is the percentage of cluster heads into the network, $r$ is the number of current cluster heads and $G$ is the group of nodes that have not been cluster head in a previous time. In this way the protocol allows the correct distribution of energy consumption in each node.

Fig. 1 shows how the network is organized in clusters, where nodes with a blue circle are the cluster heads.

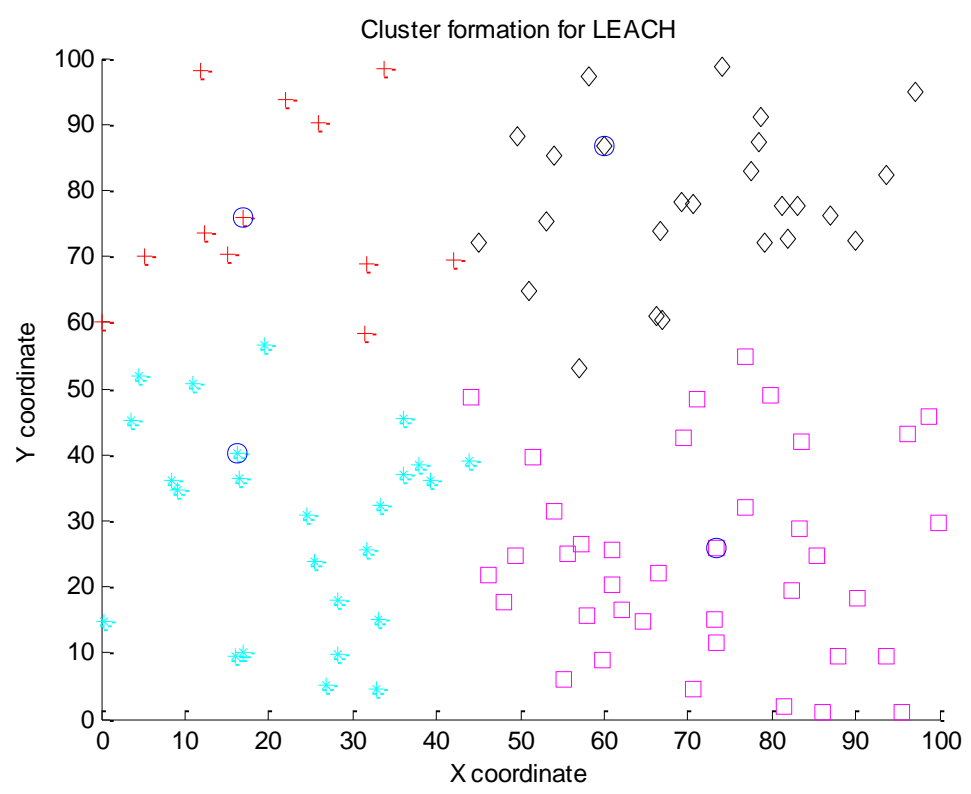

Fig. 1. Network organized in cluster for LEACH 
Effect of the error estimation of nodes in the cluster formation phase in wireless sensor networks ...

\section{Proposed Clustering Scheme}

In this work, a new clustering protocol based on the well-known LEACH protocol is proposed and studied. This clustering protocol named LEACH-MOD, works as follows: nodes are chosen to be cluster heads or cluster members only based on the order of arrival of nodes. Specifically, in the cluster formation phase, nodes transmit in each time slot according to a geometric process with probability $\tau$. In any given time slot, if there are two or more transmissions, a collision occurs and non of the transmitted packets are received successfully and they have to be retransmitted. On the other hand, if only one packet is transmitted in a time slot, it is considered to be successful and all the nodes in the network recognize such transmission. Building on this, the LEACH-MOD scheme considers that the first $N_{C H}$ nodes that successfully transmit their packet become cluster heads while the rest become cluster members. The effect of the number of $\mathrm{CHs}$ on the performance of the system is investigated in a subsequent section.

Fig. 2 shows how the network is organized in clusters, where the nodes with a blue circle are the cluster heads. We can see that the position of each node and the number of nodes per cluster is different from Fig. 1 because both schemes have a random process with uniform distribution.

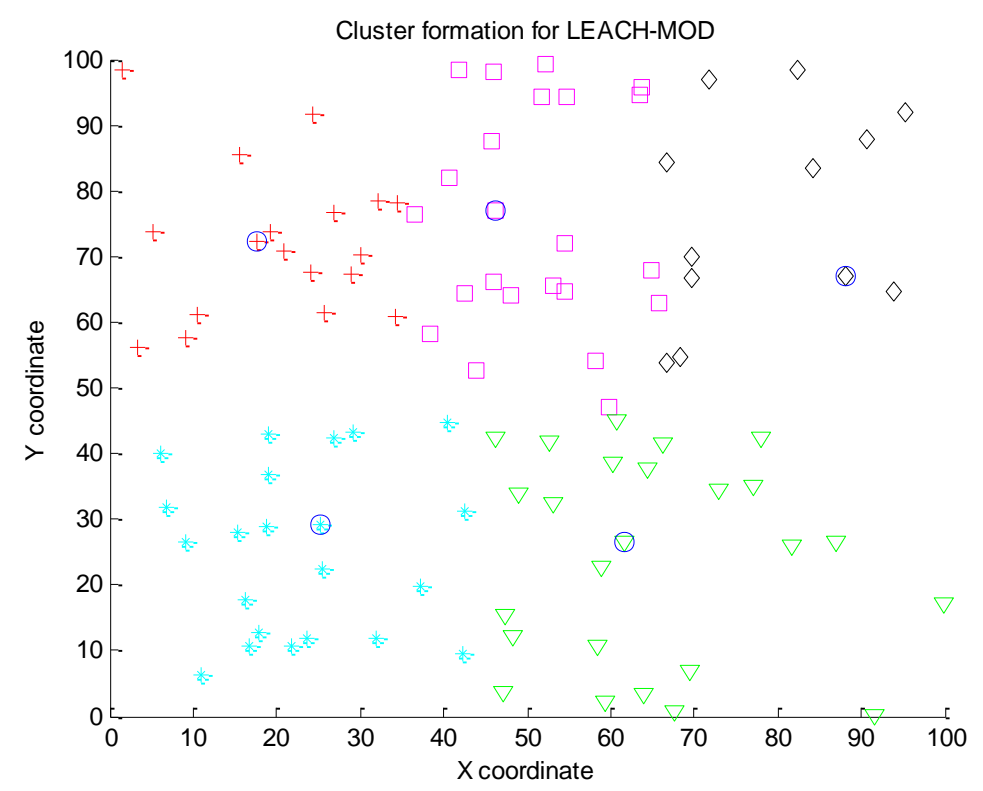

Fig. 2. Network organized in cluster for LEACH-MOD 
The main reason to consider this modification to the LEACH protocols is as follows: As mentioned before, in the LEACH protocol, each node calculates its transmission probability according to the nodes that have been $\mathrm{CHs}$ before. As such, nodes need to calculate this transmission probability throughout the operation of the networks, which entails extra energy consumption and increases complexity. On the other hand, the LEACH-MOD strategy does not require this transmission probability computation. It is important to mention that the LEACH protocol ensures that no node can become $\mathrm{CH}$ in successive rounds. This cannot be guaranteed by our proposed protocol. However, since nodes transmit according to a geometric process with parameter $\tau$, the probability that a node is elected as a $\mathrm{CH}$ in consecutive rounds is very low. Also note that neither LEACH nor LEACH-MOD protocols ensure a uniform distribution of clusters in the system area.

On a more detailed study on the effect of the transmission probability in LEACHMOD, note that when the number of nodes with a pending transmission is relatively high, the use of a high value of $\tau$ causes a high amount of collisions inside the cluster formation process, entailing a quick energy drain form all nodes in the system. On the other hand, the use of a low transmission probability in the same scenario entails a lower number of collisions. However, as nodes successfully transmit their control packet, the number of pending transmissions decreases accordingly. Hence, the low value of $\tau$ is no longer adequate when only a few nodes remain to transmit their packet since a high idle transmission period occurs. Building on this observation, it is clear that the value of the transmission probability has to vary according to the number of remaining nodes in the cluster formation procedure. As such, the adaptive transmission strategy is proposed.

\subsection{Adaptive Transmission}

As mentioned before, in the adaptive transmission probability strategy, the value of $\tau$ varies according to the number of nodes with a pending transmission. Hence, the transmission probability of each node depends on the inverse of the number of neighbor nodes. In this way, with a high number of contending nodes, the transmission probability is low, and viceversa. From this, each node computes the value of $\tau$ as follows:

$$
\tau_{\boldsymbol{A}}=\frac{1}{\boldsymbol{i}}
$$

Where $i$ is the number of remaining nodes in the cluster formation phase. As it can be seen, in order to calculate the value of $\tau$, it is required that each node estimates the number of nodes with a pending transmission. Also, the sink node, which does not have energy restriction issues, can perform this estimation and broadcast it to the rest of the nodes in the system. In this work, we consider the second approach. 
Effect of the error estimation of nodes in the cluster formation phase in wireless sensor networks ...

This estimation can be done by detecting each successful transmission and assuming that the number of nodes in the system has not changed, i.e., that all initial nodes are still correctly functioning. However the successful transmission detection is not error-free in a wireless environment due mainly to fading, interference and noise. As such, we propose to study the effect of error estimation on the performance of the adaptive probability scheme. Specifically, we consider that two estimation errors can occur in such environment: false positive and false negative estimation. The latter corresponds to the case when the sink node does not detect a successful transmission while the former corresponds to the case when the sink node detects a successful transmission but in reality no such transmission occurred. As such, the estimation of $\tau$ can have a higher (in case of false negative estimation) or lower value (in case of false positive estimation) than the real value $i$. In this work, we assume that either false positive and false negative estimation errors are equally probable to happen. Hence, a uniform error distributed in the range of $(-\varepsilon, \varepsilon)$ is considered. Consider that this assumption may not be accurate since the error can have different distribution or false negative and positive estimation may not be equally probable. However, the use of an accurate error model is outside the scope of this work and we leave it for future research.

From the previous discussion, we consider that the transmission probability in the cluster formation phase is now calculated as:

$$
\tau_{A}=\frac{1}{i+\varepsilon}
$$

Note that as in ( 2 ), the transmission probability varies in each time slot according to the estimated outcome of the previous time slot. As a particular case, when the estimation error is such that $\mathrm{i}+\epsilon \leq 0$, the sink node broadcast the value of $\boldsymbol{\tau}_{\boldsymbol{A}}=0.9$ in order to avoid any operation malfunctions. Due to the fact that $\boldsymbol{\tau}_{\boldsymbol{A}}$ and $\boldsymbol{\tau}$ are probabilities, they only can have values between 0 and 1 , being 1 the maximum value where no more nodes are waiting to transmit and we can warrant a successful transmission to particular node; in the case when those probabilities have a minimum value represents that a big number of nodes are pending to transmit.

\section{Numerical Results}

In this section, we show some relevant numerical results. The main performance parameters considered in this section are the average cluster formation time and average energy consumption. Fig. 3 shows the average energy consumption in the system for different number of nodes in the network and different error estimation range $\varepsilon$. It can be seen that the use of the LEACH-MOD with the adaptive transmission scheme achieves lower energy consumption than the LEACH protocol. This is because in the LEACH-MOD the number of collisions and idle listening periods are reduced compared to the LEACH protocol. Similarly, the average cluster 
formation time is lower in the LEACH-MOD compared to the LEACH protocol as shown in Fig. 4. As expected, the energy consumption and cluster formation time increase as the number of nodes increases.

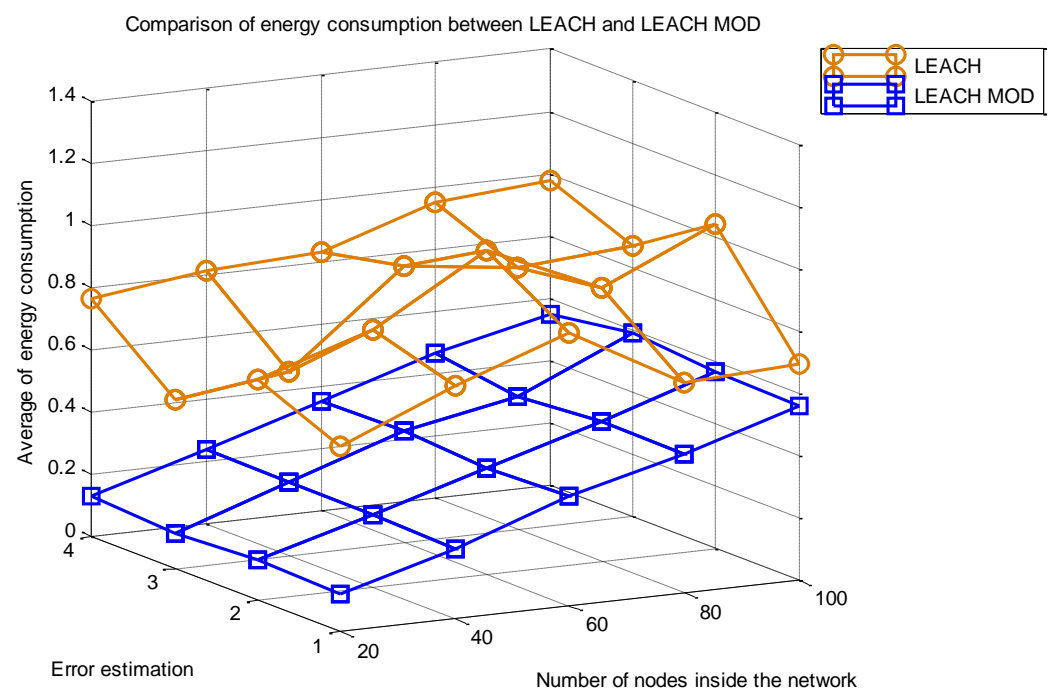

Fig. 3. Average cluster energy consumption under an adaptive probability of transmission

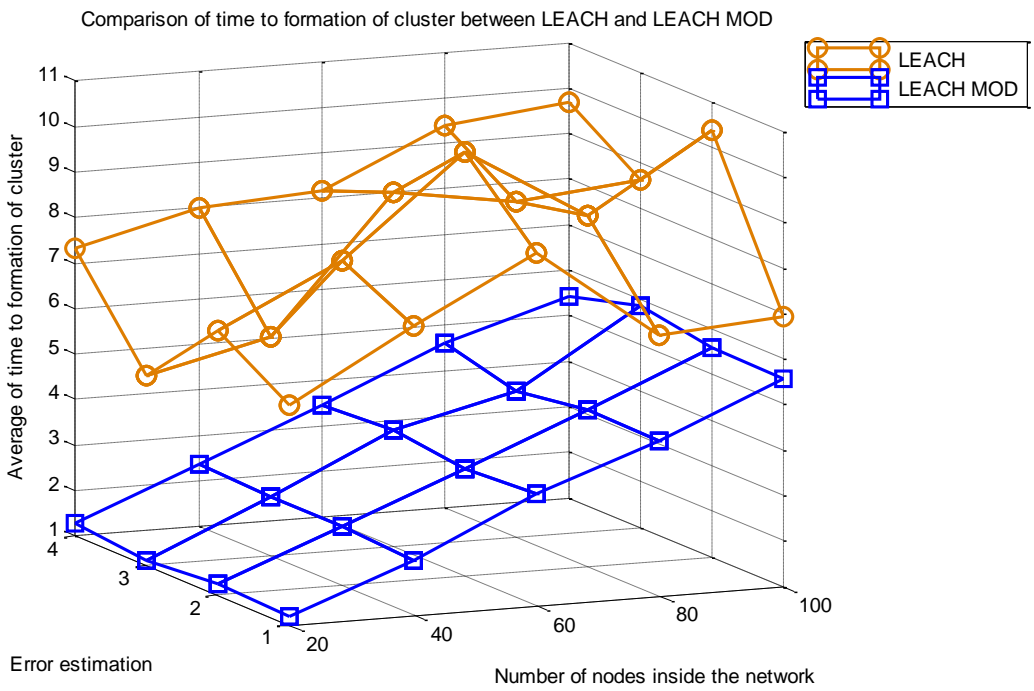

Fig. 4. Average cluster formation time under an adaptive probability to transmission 
Effect of the error estimation of nodes in the cluster formation phase in wireless sensor networks ...

From these results, it can be seen that the effect of the error estimation is not high. The rationale behind this counterintuitive result is as follows: since a uniformly distributed estimation error is considered, in a given time slot a false positive error can occur while in the following slot a false negative error can happen with the same probability, cancelling out the effect of the error estimation. Hence, different estimation error distribution could be considered in a future work.

\section{Conclusions}

In this work, the LEACH-MOD protocol under an adaptive transmission probability is proposed and studied. From the results it is clear that a simple $\mathrm{CH}$ selection, like the LEACH-MOD protocol, can achieve a good system performance because the energy consumption and formation cluster time is lower than LEACH. Hence, energy consumption and complexity are reduced. Also, the use of the adaptive transmission strategy is of major importance to reduce energy consumption at the cluster formation phase. It is shown that a constant transmission probability entails a high amount of collisions and/or idle listening periods. However, the complexity of the adaptive scheme requires potential transmissions estimation. The design and proposal of an estimation algorithm is outside the scope of this paper due to its complexity.

However, we are interested on evaluating the system performance considering an estimation error. Furthermore, we proposed to study the effect of a uniformly distributed error in the range of $[-\varepsilon, \varepsilon]$ that models both the false positive and false negative estimation errors. From the presented results, it can be seen that the effect of such error is very low. Indeed, by considering a uniformly distributed error, the effect of a false negative error is cancelled out by the false positive errors. As such, following this line of research, different error distribution must be considered for a practical system implementation.

\section{References}

1. Handy, M. J., Haase, M., Timmermann, D.: Low Energy Adaptive Clustering Hierarchy with Deterministic Cluster-Head Selection. 4th International Workshop on Mobile and Wireless Communications Network, 2002.

2. Rabiner ,W., Chandrakasan A., Balakrishnam, H.: Energy-Efficient Comunication Protocol for Wireless Microsensor Networks. Massachusetts institute of Technology Cambridge, Massachusetts, USA, 2000.

3. Kredo, K.: Medium Access Control in Wireless Sensor Networks. University of California, California, USA.

4. Lee H.: Towards a General Wireless Sensor Network Platform. IEEE, Taiwan, ROC, 2012.

5. Johnson, D.B., Maltz, D.A.: Protocols for Adaptive Wireless and Mobile Networking. IEEE Personal Communications, vol. 3, no. 1, 1996. 
6. Owojaiye G., Sun, Y.: Focal design issues affecting the deployment of wireless sensor networks for intelligent transport. IEEE, UK., 2012.

7. Ruizhong, L., Zhi, W., Youxian, S: Energy Efficient Medium Access Control Protocols for Wireless Sensor Networks and Its State-of-Art. National Laboratorv of Industrial Control Technology, Institute of Modem Control Engineering, Hangzhou, China, 2004.

8. Spyropoulos, T., Psounis, K., Raghavendra, C.: Performance Analysis of Mobility-assisted Routing. Department of Electrical Engineering, USC, Florence, Italia, 2006. 